\title{
Reductive algebraic groups
}

\author{
By \\ Hideyasu Suminiro \\ (Communicated by Professor Nagata, May 10, 1971)
}

\section{o. Introduction.}

We shall consider linear algebraic groups defined over an algebraically closed field $k$ with an arbitrary characteristic $p$. For the simplicity, we shall call them algebraic groups. Let $G$ be an algebraic group and let $V$ be a finite dimensional $k-G$-rational module. If $G$ fixes a non-zero vector $e_{0}$ of $V$, then the associated representation of $G$ is called an $M$-representation (or the representation of $M$-type), and $e_{0}$ is called the associated fixed point. Extend $e_{0}$ to a basis $\left\{e_{0}, e_{1}, \ldots, e_{n}\right\}$ of $V$. Then, we have a matric representation $\rho^{\prime}: G \rightarrow G L(V)$ under the basis $\left\{e_{0}, e_{1}, \ldots, e_{n}\right\}$ of the following form

$$
\rho^{\prime}(g)=\left(\begin{array}{cc}
1 & u(g) \\
0 & \\
\vdots & \rho(g) \\
0 &
\end{array}\right)
$$

where $u(g)$ is a $(1 \times n)$-matrix and $\rho(g)$ is an $(n \times n)$-matrix. Through this representation of $G, G$ acts rationally on the projective space $P_{n}$ and fixes a point $e_{0}=\left(\begin{array}{c}1 \\ 0 \\ \vdots \\ 0\end{array}\right)$. Therefore, $G$ acts rationally on the polynomial ring $k\left[X_{0}, \ldots, X_{n}\right]$ in the following way;

$$
X_{0}^{g}=X_{0}+\sum_{i=1}^{n} u_{i}(g) X_{i}
$$




$$
X_{i}^{g}=\sum_{j=1}^{n} \rho_{i j}(g) X_{j} \quad(1 \leq i \leq n)
$$

Under the above notation, the following conditions are equivalent to each other.

(a) For any $M$-representation $\rho^{\prime}: G \rightarrow G L(n+1, k)$, there exists a $G$-invariant monic polynomial with respect to $X_{0}$.

(b) For any $M$-representation $\rho^{\prime}: G \rightarrow G L(n+1, k)$, there exists a $G$-stable hypersurface in $P_{n}$ which does not go through the associated fixed point $e_{0}$ (i.e. there exists a $G$-stable affine open subset in $P_{n}$ which contains $\left.e_{0}\right)$.

(c) Let $R$ and $R^{\prime}$ be any $G$-rational $k$-algebras such that there is a surjective $G$-algebra homomorphism $\varphi: R \rightarrow R^{\prime}$. Then, for any $G$ invariant element $x$ of $R^{\prime}$, there exists a $G$-invariant element $y$ of $R$ and a positive integer $m$ such that $\varphi(y)=x^{m}$.

An algebraic group $G$ which satisfies the above equivalent conditions is called geometrically reductive (Seshadri [12]). In connection with the construction of moduli space of curves over an arbitrary field, D. Mumford [5] conjectured that a connected reductive algebraic group is geometrically reductive. Moreover, this conjecture concerns with the 14th problem of Hilbert (Nagata [9]), the moduli space of stable vector bundles over a non-singular complete curve (Seshadri [12]) and quotient homogeneous spaces. In this paper, we shall prove the followings: Let $G$ be a connected reductive algebraic group. Then, for any $M$-representation $\rho^{\prime}: G \rightarrow G L(n+1, k)$, there exists a $G$-stable closed subset in $P_{n}$ (which may not be a hypersurface) which does not contain the associated fixed point. Furthermore, we shall discuss one question "Does this property characterize reductive algebraic groups?" and consider one application.

The author likes to express his sincere thanks to Professor M. Nagata and Professor H. Hijikata for many valuable comments and discussions. 


\section{Geometrically reductive groups and semi-reductive groups}

Definition 1.1. Let $G$ be an algebraic group. If, for any $M$ representation $\rho^{\prime}: G \rightarrow G L(n+1, k)$, there exists a $G$-stable closed subset in $\mathbf{P}_{n}$ which does not contain the associated fixed point, then $G$ is called semi-reductive algebraic group.

Our aim of this section is to prove that a connected reductive algebraic group is semi-reductive. We shall prepare some lemmas for the purpose.

Lemma 1.2. Let $G$ be a connected algebraic group and let $B$ and $T(B) T)$ be a Borel subgroup of $G$ and a maximal torus of $G$ respectively. If $\rho^{\prime}: G \rightarrow G L(n+1, k)$ is an M-representation, then there is a matrix $S(\in G L(n+1, k))$ such that $\rho^{\prime \prime}=S \rho^{\prime} S^{-1}$ satisfies the following conditions.

(1) $\rho^{\prime \prime}$ is an M-representation of $G$.

(2) $\rho^{\prime \prime}(B)=\left\{\rho^{\prime \prime}(b) \mid b \in B\right\}$ consists only of upper triangular matrices and $\rho^{\prime \prime}(T)=\left\{\rho^{\prime \prime}(t) \mid t \in T\right\}$ consists only of diagonal matrices.

Proof. Put $\rho^{\prime}=\left(\begin{array}{ll}1 & u \\ 0 & \rho\end{array}\right) . \quad \rho$ is a representation of $G$.

Hence, there is a matrix $\bar{S}_{1}(\in G L(n, k))$ such that $\bar{\rho}=\bar{S}_{1} \rho \bar{S}_{1}^{-1}$ satisfies that $\bar{\rho}(B)$ (or $\bar{\rho}(T)$ respectively) are upper triangular matrices

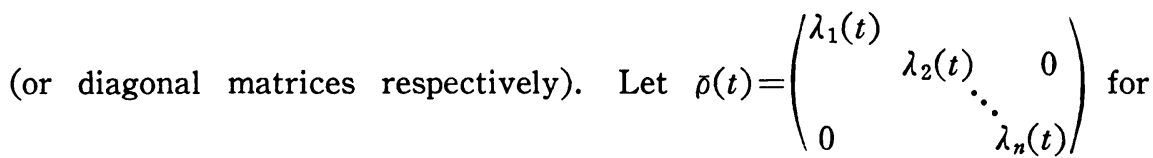

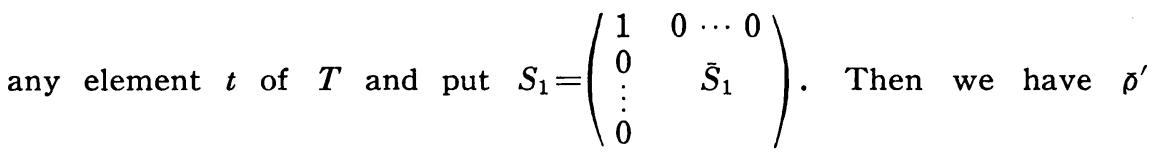
$=S_{1} \rho^{\prime} S_{1}^{-1}=\left(\begin{array}{cc}1 & u \bar{S}_{1}^{-1} \\ 0 & \bar{S}_{1} \rho \bar{S}_{1}^{-1}\end{array}\right)=\left(\begin{array}{cc}1 & \bar{u} \\ 0 & \bar{\rho}\end{array}\right)$, where $\bar{u}=u \bar{S}_{1}^{-1}$. Let $Q_{i}$ be the connected component of $\operatorname{Ker} \lambda_{i}$ at the unit element and $I=\left\{i \mid Q_{i}=T\right\}$. Take an element $t_{0}$ of $T$ such that the closed subgroup of $T$ which 
contains $t_{0}$ is $T$ itself.

Put

$$
a_{i}=\left\{\begin{array}{cc}
\frac{-u_{i}\left(t_{0}\right)}{\lambda_{i}\left(t_{0}\right)-1} & (i \notin I) \\
0 & (i \in I)
\end{array}\right.
$$

and $S_{2}=\left(\begin{array}{cc}1 & a_{1}, \ldots, a_{n} \\ 0 & E_{n} \\ \vdots & \\ 0 & \end{array}\right)$, where $E_{n}$ is the unit matrix.

Then $S=S_{2} S_{1}$ satisfies the Lemma 1.2 .

q.e.d.

The following Lemma 1.3. is a key Lemma to prove that a connected reductive algebraic group is semi-reductive.

Lemma 1.3. Let $G$ be a connected algebraic group, $\rho^{\prime}: G \rightarrow$ $G L(n+1, k)$ an M-representation and let $B$ be a Borel subgroup of $G$. The following conditions are equivalent to each other.

(1) There exists a G-stable closed subset in $\mathbf{P}_{n}$ which does not contain the associated fixed point $e_{0}$.

(2) There exists a point $x\left(\neq e_{0}\right)$ in $\mathbf{P}_{n}$ which is a $B$-fixed point

(3) Put $\rho^{\prime}=\left(\begin{array}{ll}1 & u \\ 0 & \rho\end{array}\right)$. For each element $g$ of. $G$, put $H_{g}$ $=\left\{y \in \mathbf{P}_{n-1} \mid u(g) y=0\right\} \quad$ (this forms a hyperplane in $\left.\mathbf{P}_{n-1}\right)$. Then, $\bigcap_{b \in B^{u}} H_{b} \neq \phi\left(B^{u}\right.$ being the unipotent part of $\left.B\right)$

Proof. The equivalence of (1) and (2) is obvious. (2) $\rightarrow$ (3). Let $x\left(\neq e_{0}\right)$ be a $B$-fixed point. Put $x=\left(\begin{array}{l}x_{0} \\ x^{\prime}\end{array}\right)$, where $x_{0}$ is an element of $k$ and $x^{\prime}(\neq 0)$ is an $(n \times 1)$-matrix. From the hypothesis, there is a rational character $\lambda: B \rightarrow k^{*}$ such that

$$
\left(\begin{array}{cc}
1 & u(b) \\
0 & \rho(b)
\end{array}\right)\left(\begin{array}{c}
x_{0} \\
x^{\prime}
\end{array}\right)=\lambda(b)\left(\begin{array}{c}
x_{0} \\
x^{\prime}
\end{array}\right) \text { for any element } b \text { of } B
$$

Hence, $x_{0}+u(b) x^{\prime}=\lambda(b) x_{0}$. But $\lambda(b) \equiv 1$ for any element $b$ of 
$B^{u}$. Thus $x^{\prime} \in \bigcap_{b \in B^{u}} H_{b}$ and so $\bigcap_{b \in B^{u}} H_{b}$ is not empty. (3) $\rightarrow(2)$. Let $T$ be a maximal torus of $G$ contained in $B$. By virtue of Lemma 1.2, we may assume that the $M$-representation $\rho^{\prime}=\left(\begin{array}{ll}1 & u \\ 0 & \rho\end{array}\right)$ satisfies the conditions (1) and (2) of Lemma 1.2. For any elements $b, b^{\prime}$ of $B^{u}$ and $t$ of $T$,

$$
\begin{aligned}
& u\left(b b^{\prime}\right)=u\left(b^{\prime}\right)+u(b) \rho\left(b^{\prime}\right) \\
& u\left(t^{-1} b t\right)=u(t)+u\left(t^{-1} b\right) \rho(t)=u\left(t^{-1} b\right) \rho(t) \\
& \quad=\left(u(b)+u\left(t^{-1}\right) \rho(b)\right) \rho(t) \\
& \quad=u(b) \rho(t) .
\end{aligned}
$$

Put $H=\bigcap_{b \in B^{u}} H_{b}(\neq \phi)$. For any element $x^{\prime}$ of $H$, we have that

$$
\begin{aligned}
& 0=u\left(b b^{\prime}\right) x^{\prime}=u\left(b^{\prime}\right) x^{\prime}+u(b) \rho\left(b^{\prime}\right) x^{\prime}=u(b) \rho\left(b^{\prime}\right) x^{\prime} \\
& 0=u\left(t^{-1} b t\right) x^{\prime}=u(b) \rho(t) x^{\prime}
\end{aligned}
$$

Hence, $H$ is a $B$-stable linear subvariety of $\mathbf{P}_{n-1}$. By the theorem of Lie-Kolchin, there exists a $B$-fixed element $x^{\prime}(\neq 0)$ in $H$. Put $x$ $=\left(\begin{array}{c}0 \\ x^{\prime}\end{array}\right)$. Then we have,

$$
\left(\begin{array}{cc}
1 & u(b) \\
0 & \rho(b)
\end{array}\right)\left(\begin{array}{l}
0 \\
x^{\prime}
\end{array}\right)=\left(\begin{array}{l}
u(b) x^{\prime} \\
\rho(b) x^{\prime}
\end{array}\right)=\left(\begin{array}{l}
0 \\
\rho(b) x^{\prime}
\end{array}\right)
$$

for any element $b$ of $B$, because $u(b) x^{\prime}=0$ for any element $b$ of $B$. Thus, $x$ is a $B$-fixed point which is different from the associated fixed point.

q.e.d.

Corollary 1.4. For a connected solvable algebraic group $G$, the following conditions are equivalent to each other;

(1) $G$ is geometrically reductive.

(2) $G$ is reductive.

(3) $G$ is semi-reductive. 
Proof. The equivalence of (1) and (2) is obvious. We have only to prove that (3) implies (1). Let $\rho^{\prime}: G \rightarrow G L(n+1, k)$ be an $M$ representation of $G$. By virtue of Lemma $1.3, \rho^{\prime}$ is quivalent to an $M$-representation of type $\left(\begin{array}{cccc}1 & 0 & \ldots & 0 \\ 0 & & & \\ \vdots & & * \\ 0 & & \end{array}\right)$. Therefore, there exists a $G$ stable hyperplane which does not contain the associated fixed point.

q.e.d.

Corollary 1.5. Let $G$ be a connected algebraic group and let $N$ be a closed connected subgroup of $G$ such that $B_{N}^{u}=B^{u}\left(B_{N}^{u}\right.$ being the unipotent part of a Borel subgroup of $N$.) If $N$ is semi-reductive, then $G$ is semi-reductive. In particular, if $N$ is a closed connected normal subgroup of $G$, if $G / N$ is a torus group and if $N$ is semi-reductive, then $G$ is semi-reductive.

Proof. The first part follows directly from Lemma 1.3. Since $G / N$ is a torus group, $B_{N}^{u}=B^{u}$ and the second part is obvious. q.e.d.

Next we shall prove some propositions about semi-reductive groups.

Proposition 1.6. (1) Let $G$ and $G^{\prime}$ be algebraic groups. If there is a surjective homomorphism from $G$ to $G^{\prime}$ and if $G$ is semi-reductive, then $G^{\prime}$ is semi-reductive.

(2) Let $G$ be a connected algebraic group and let $N$ be a closed connected normal subgroup of $G$. If $N$ is geometrically reductive and if $G / N$ is semi-reductive, then $G$ is semi-reductive.

Proof. (1) is obvious. (2). Let $\rho^{\prime}: G \rightarrow G L(n+1, k)$ be an $M$ representation of $G$. There is an $N$-invariant monic homogeneous polynomial $F\left(x_{0}, \ldots, x_{n}\right)$ with respect to $x_{0}$ because $N$ is geometrically reductive. Put $V=\sum_{g \in G} F^{g} k$. Then $V$ is a finite dimensional $G / N$. rational module. Put $W=V \cap\left(\sum_{i \geq 1} x_{i} \cdot k\left[x_{0}, \ldots, x_{n}\right]\right)$. Then $V=F \cdot k \oplus W$ 
and $V$ gives an $M$-representation of $G / N$. Let a be the ideal generated by $\left\{F^{g}\right\}_{g \in G}$ in $k\left[x_{0}, \ldots, x_{n}\right]$. $a$ is a $G$-stable ideal. If the associated closed subset $V(\mathfrak{a})$ in $\mathbf{P}_{n}$ is non-empty, then $V(\mathfrak{a})$ is a $G$-stable closed subset which does not contain the associated fixed point. Thus, we may assume that $V(\mathfrak{a})$ is empty. Let $\left\{F_{1}, \ldots, F_{m}\right\}$ be a basis of $W$ and $F_{0}=F$. Then the map $\varphi: \mathbf{P}_{n} \ni x=\left(x_{0}: \ldots: x_{n}\right) \rightarrow\left(F_{0}(x): \ldots: F_{m}(x)\right) \in \mathbf{P}_{m}$ is a non-constant morphism. Hence, $\operatorname{dim}(\operatorname{Im} \varphi)=n . F_{i}\left(x^{g}\right)=F_{i}(x)$ $(0 \leq i \leq m)$ for any point $x$ of $\mathbf{P}_{n}$ and any $g(\in N)$, because $F_{i}$ is $N$ invariant. Thus the orbit $N(x)$ of $x$ is contained in $\varphi^{-1}(\varphi(x))$ for any point $x$ of $P_{n}$. By the dimension theorem of morphisms, $N(x)=x$ for a general point $x$ of $P_{n}$. Therefore, $\rho^{\prime} \mid N$ is a unit representation and so $\rho^{\prime}$ is an $M$-representation of $G / N$. Hence, there exists a $G$ stable closed subset in $\mathbf{P}_{n}$ which does not contain the associated fixed point.

q.e.d.

Remark 1.7. Let $G$ be a connected algebraic group and let $N$ be a closed normal subgroups of $G$ (not necessarily connected). If $N$ is completely reducible (i.e. every rational representation of $N$ is completely reducible.) and if $G / N$ is semi-reductive, then we can prove that $G$ is semi-reductive by the same method as in the proof of Proposition 1.1 (2).

Proposition 1.8. Let $G$ be a connected semi-simple algebraic group. Then $G$ is semi-reductive.

Proof. Let $B$ and $T$ be a Borel subgroup of $G$ and a maximal torus of $G$ contained in $B$ respcetively. Let $\rho^{\prime}: G \rightarrow G L(n+1, k)$ be an $M$-representation of $G$ which satisfies the conditions of Lemma 1.2. Furthermore, Let $r$ be $\operatorname{dim} T, \Sigma=\{\alpha\}$ (or $\Sigma_{0}=\left\{\alpha_{1}, \ldots \alpha_{r}\right\}$ respectively) be the positive root system of $G$ (or fundamental root system respectively) and let $X$ be the rational character group of $T$. Then $\left\{\alpha_{1}, \ldots, \alpha_{r}\right\}$ is a basis of $X \underset{z}{\otimes} Q$ over $Q$ ( $Q$ being the rational number 
field.). Put $\rho^{\prime}=\left(\begin{array}{cc}1 & u \\ 0 & \rho\end{array}\right)$ and $\rho^{\prime}(t)=\left(\begin{array}{ccc}1 & 0 \ldots & 0 \\ 0 & \lambda_{1}(t) \\ \vdots & & \ddots \\ 0 & & \lambda_{n}(t)\end{array}\right)$ for each element $t$ of $T$. For any positive root $\alpha$, there is a one-parameter subgroup $\tau_{\alpha}: k \rightarrow P_{\alpha}$ such that $t \tau_{\alpha}(x) t^{-1}=\tau_{\alpha}(\alpha(t) x)(t \in T, x \in k)$. Each element $b$ of $B^{u}$ can be written uniquely in the following way; $b=\prod_{\alpha \in \Sigma} \tau_{\alpha}\left(x_{\alpha}\right)$ $\left(x_{\alpha} \in k\right)$. Hence, we have that

$u_{i}(b)=\sum_{m=\left(m_{\alpha}\right)} c_{m}^{i} \Pi x_{\alpha}^{m_{\alpha}}$ where $c_{m}^{i}$ are elements of $k$ and $m_{\alpha}$ are nonnegative integers. From this, we have the following;

$$
\begin{gathered}
u_{i}\left(t b t^{-1}\right)=u_{i}\left(\prod_{\alpha \in \Sigma} \tau_{\alpha}\left(\alpha(t) x_{\alpha}\right)\right)=\sum_{m=\left(m_{\alpha}\right)} c_{m}^{i} \prod \alpha(t)^{m_{\alpha}} \prod x_{\alpha}^{m_{\alpha}} \\
u_{1}\left(t b t^{-1}\right)=\lambda_{i}^{-1}(t) u_{i}(b)=\lambda_{i}^{-1}(t) \sum_{m=\left(m_{\alpha}\right)} c_{m}^{i} \prod x_{\alpha}^{m_{\alpha}} .
\end{gathered}
$$

Thus, if some $c_{m}^{i}$ is not equal to zero, then $-\lambda_{i}=\sum_{\alpha} m_{\alpha} \cdot \alpha$. Put $\lambda_{i}=\sum_{k=1}^{r} r_{i k} \cdot \alpha_{k}(1 \leq i \leq n)$, where $r_{i k}$ are rational numbers. If some $r_{i k}$ is positive, then $u_{i} \equiv 0$ on $B^{u}$. Since $G$ is semi-simple, $\sum_{i=1}^{n} \lambda_{i}=\sum_{k=1}^{r}\left(\sum_{i=1}^{n} r_{i k}\right) \alpha_{k}$ $=0$, and $\sum_{i=1}^{n} r_{i k}=0$ for all $k$. If each $r_{i k}$ is not positive, then each $r_{i k}$ is equal to zero. In this case, $\rho^{\prime}$ is a unit representation. By the above argument, we can have that $u_{i} \equiv 0$ for some $i$ on $B^{u}$. This and Lemma 1.3 imply that there exists a $G$-stable closed subset which does not contain the associated fixed point.

q.e.d.

Theorem 1.9. Let $G$ be a connected reductive group. Then $G$ is semi-reductive.

Proof. By vitue of Corollary 1.5 or Proposition 1.6 (2), we can easily prove Theorem 1.9 .

Problem 1. Let $G$ be a connected reductive algebraic group and let $\rho^{\prime}: G \rightarrow G L(n+1, k)$ be an $M$-representation of $G$. Assume that $X$ is a $G$-stable closed subvariety in $\mathbf{P}_{n}$ which contains the associated fixed point and dimension of $X$ is greater than one. Then, does there exist 
a $G$-stable closed proper subset $Y$ of $X$ which does not contain the associated fixed point?

Remark 1.10. If Mumford conjecture is true, then we can easily prove that problem 1 is true.

\section{Reductive algebraic groups.}

In this section, we shall consider the relation between reductive algebraic groups and semi-reductive algebraic groups.

Lemma 2.1. (Steinberg [13]) Let $G^{\prime}$ be a connected simple algebraic group and let $(G, \pi)$ be a central extension of finite type of $G^{\prime}$ $\left(\pi ; G \rightarrow G^{\prime}\right.$ is a surjective homomorphism, Ker $\pi$ is a central subgroup of $G$ and order of any element of $\operatorname{Ker} \pi$ is bounded). Then there is a central extension $\left(\Gamma, \pi^{\prime}\right)$ of $G^{\prime}$ such that

(1) There is a group homomorphism $\sigma: I \rightarrow G$

(2) $\Gamma=[\Gamma, \Gamma]$ and $\pi^{\prime}: \Gamma \rightarrow G^{\prime}$ is an isogeny.

(3) The following diagram is commutative

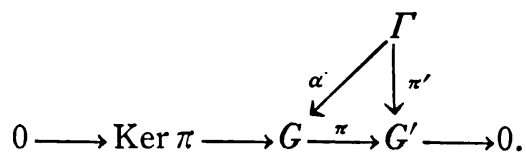

We shall use this Lemma 2.1 to prove the following.

Proposition 2.2. Let $G$ be a connected algebraic group and let $R$ be its radical. If $R=R^{u}, \operatorname{dim} R \geq 1$ and if $R$ is a central subgroup of $G$, then $G \neq[G, G]$.

Proof. If characteristic of $k$ is zero, our assertion is obvious by the Levi decomposition and we may assume that characteristic of $k$ is positive. We shall prove Proposition 2.2 by the induction on $\operatorname{dim} G$. If $\operatorname{dim} G=1$, then $G=R=R^{u}$ is a commutative group, whence Proposition 2.2 is true. Assume that $\operatorname{dim} G>1$ and put $G^{\prime}=G / R$. If $G^{\prime}$ is a 
simple algebraic group, then $G=\sigma(\Gamma) \cdot R=R \cdot \sigma(\Gamma)$ by virtue of Lemma 2.1. Thus, $[G, G]=[\sigma(\Gamma), \sigma(\Gamma)]=\sigma(\Gamma) \neq G$. If $G^{\prime}=G / R$ is not simple, then $G^{\prime}=G_{1}^{\prime} \cdot G_{1}^{\prime}$, where $G_{1}^{\prime}$ is a closed normal simple subgroup, $G_{2}^{\prime}$ is a closed normal semi-simple subgroup of $G^{\prime}$ and where $\left(G_{1}^{\prime} \cap G_{2}^{\prime}\right)$ is a finite group. Furtheoremore $G_{1}^{\prime}$ commutes with $G_{2}^{\prime}$. Let $\pi: G \rightarrow G^{\prime}$ $=G / R$ be a canonical homomorphism and let $H$ be the connected component of $\pi^{-1}\left(G_{2}^{\prime}\right)$ at the unit element. Then, $\operatorname{dim} H<\operatorname{dim} G$ and $R$ is the radical of $H$. Hence the induction hypothesis implies that $H \neq[H, H]$. On the other hand, $H=R \cdot[H, H]$. Thus we have that $R \nsubseteq[H, H]$. Put $G^{\prime \prime}=G /[H, H] \quad$ and $\quad \mathrm{R}^{\prime \prime}=R \cdot[H, H] /[H, H]$ $=H /[H, H]$. Then $R^{\prime \prime}$ is the radical of $G^{\prime \prime}, \operatorname{dim} R^{\prime \prime} \geq 1$ and $G^{\prime \prime} / R^{\prime \prime}$ $\cong G / H$. Since $G^{\prime \prime} / R^{\prime \prime}$ is simple, we have that $G /[H, H] \neq[G /[H, H]$, $G /[H, H]]=[G, G] /[H, H]$. Therefore we have that $G \neq[G, G]$.

q.e.d.

Corollary 2.3. Let $G$ be a connected algebraic group and $R$ be the radical of $G$. If $G=[G, G]$ and $R$ be a central subgroup of $G$, then $G$ is semi-simple.

Proof. Put $R=R^{u} \cdot R^{s}$ where $R^{u}$ (or $R^{s}$ ) is the unipotent part of $R$ (or the semi-simple part of $R$ respectively). ( $R^{s} \cap[G, G]$ ) is a finite group, whence $R^{s}=(e)$. If $\operatorname{dim} R^{u}=\operatorname{dim} R \geq 1$, then $G \neq[G, G]$ by virtue of Corollary 2.3. Therefore $R=R^{u}=(e)$.

By virtue of Proposition 2.2, we can show a necessary and sufficient condition for semi-reductive algebraic groups to be reductive.

Theorem 2.4. Let $G$ be a connected algebraic group. The following conditions are equivalent to each other.

(1) $G$ is reductive.

(2) $G$ is semi-reductive and the unipotent radical of $G$ is a central subgroup. 
Proof. (1) $\rightarrow$ (2) is obvious. We shall prove (2) $\rightarrow$ (1) by the induction on $\operatorname{dim} G$. Let $R$ be the radical of $G$ and $R^{s}$ be the semisimple part of $R$. If $\operatorname{dim} R^{s} \geq 1$, then $G / R^{s}$ is reductive by the induction hypothesis. Hence $R^{u}=(e)$. If $\operatorname{dim} R^{s}=0$ and $\operatorname{dim} R^{u} \geq 1$, then $G \neq[G, G]$ by virtue of Proposition 2.2. But this can not occur, because $G=R \cdot[G, G]$ and $G /[G, G]$ is a torus group. $\quad$ q.e.d.

We shall show another condition next.

Theorem 2.5. Let $G$ be a connected algebraic group and $R$ be the radical of $G$. The following conditions are equivalent to each other.

(1) $G$ is reductive.

(2) $G$ is semi-reductive and $\operatorname{dim} R^{u} \leq 1$.

Proof. (1) $\rightarrow$ (2) is obvious. We shall prove $(2) \rightarrow(1)$ by the induction on $\operatorname{dim} G$. If $\operatorname{dim} G=1$, then our theorem is obvious. Assuming that $\operatorname{dim} R^{u}=1$, we shall derive a contradiction. Put $\pi: G \ni g i m \rightarrow$ Int. $g \in$ Aut $_{\text {2lg.gr. }} R^{u}=k^{*}$ (Int. $g$ is the inner automorphism of $G$ by $g$ ). Furthermore, let $G^{\prime}$ be the connected component of $(\operatorname{Ker} \pi)$ at the unit element. Then $G^{\prime}$ is a closed connected normal subgroup of $G$ and $\operatorname{codim} G^{\prime} \leq 1$. Put $R^{\prime}$ to be the connected component of $\left(R \cap G^{\prime}\right)$ at the unit element. Then $R^{\prime}$ is the radical of $G^{\prime}$ and $R^{\prime u}=R^{u}$. Since $R^{\prime u}$ is a central subgroup of $G^{\prime}, R^{\prime}=R^{\prime u} . R^{\prime s}$ is commutative.

Hence $R^{\prime s}$ is a closed normal subgroup of $G$. If $\operatorname{dim} R^{\prime s} \geq 1$, then $G / R^{\prime s}$ is reductive by the induction hypothesis and so $R^{u}=(e)$. Therefore, we may assume that $R^{\prime s}=(e)$. Since $\operatorname{dim} R^{\prime u}=1, G^{\prime} \neq\left[G^{\prime}, G^{\prime}\right]$ by virtue of Proposition 2.2. On the other hand, if $\left[G^{\prime}, G^{\prime}\right] \neq e$, then $G^{\prime} /\left[G^{\prime}, G^{\prime}\right]$ is reductive and $R^{\prime u} \subseteq\left[G^{\prime}, G^{\prime}\right]$. But $G^{\prime}=R^{\prime u} \cdot\left[G^{\prime}, G^{\prime}\right]$. This is a contradiction. Hence $G^{\prime}$ is a commutative group and $G$ is solvable. By virtue of Corollary $1.4, G$ is torus group. This is also a contradiction.

q.e.d.

Next we shall prove that semi-reductive algebraic groups are reduc- 
tive in the case of characteristic zero. At first, we shall prepare two lemmas in order to prove it.

Lemma 2.6. (Mostow [6]) Let $G$ be a connected algebraic group and let $R^{u}$ be the unipotent radical of $G$. If characteristic of $k$ is zero, then for any maximal closed connected reductive subgroup $G^{\prime}$ of $G$, we have that $G=R^{u} \cdot G^{\prime}=G^{\prime} \cdot R^{u}$ (semi-direct).

Therefore, fiber space $\pi: G \rightarrow G / R^{u}$ has a global section which is a group homomorphism.

Lemma 2.7. (Birula [2]) Let $G$ be a connected algebraic group and let $H$ be a closed connected unipotent subgroup of $G$ such that $G / H$ is affine. Then, for any $k-H-$ rational module $M$, there is a $k-G-$ rational module $N$ which satisfies,

(1) $M$ is a $k-H$ - rational submodule of $N$,

and

(2) $M^{H}=N^{G}$ where $M^{H}=\left\{m \in M \mid m^{h}=m\right.$ for every element $h$ of $H\}$ and $N^{G}=\left\{n \in N \mid n^{g}=n\right.$ for every element $g$ of $\left.G\right\}$.

Now we shall prove the following.

Theorem 2.8. In the case of characteristic zero, the following conditions are equivalent to each other.

(1) $G$ is geometrically reductive.

(2) $G$ is reductive.

(3) $G$ is semi-reductive.

Proof. It is well-known that (1) and (2) are equivalent to each other (Nagata [10]). We have only to prove that (3) implies (2). We shall prove it by the induction on $\operatorname{dim} G$. If $\operatorname{dim} G=0$ or 1 , then it is obvious. If every $M$-representation of two size of $R^{u}$ is trivial, then $R_{u}$ is trivial. We shall prove that evely $M$-representation of two 
size of $R^{u}$ is trivial. Let $V=e_{0} k+e_{1} k$ be an $M$-representation module of $R^{u}$ and let $R^{u} \ni b \cdots\left(\begin{array}{ll}1 & v(b) \\ 0 & 1\end{array}\right) \in G L(2, k)$ be the associated representation. By lemma 2.7, let $W=\sum_{g \in G} e_{1}^{g} \cdot k$ and let $\left\{e_{0}, e_{1}, e_{1}^{g_{2}}, \ldots\right.$, $\left.e_{1}^{g_{m}}\right\}\left(g_{i} \in G\right)$ be a basis of $W$. For any element $b$ of $R^{u}$,

$$
\begin{aligned}
& e_{1}^{b}=v(b) e_{0}+e_{1} \\
& e_{1}^{g_{i} b}=e_{1}^{g_{i} b g_{i}^{-1} g_{i}}=v\left(g_{i} b g_{i}^{-1}\right) e_{0}+e_{1}^{g_{i}} \quad(i=2, \ldots, m) .
\end{aligned}
$$

Therefore, we have an $M$-representation $\rho^{\prime}=\left(\begin{array}{ll}1 & u \\ 0 & \rho\end{array}\right)(\in G L(m+1, k))$ of $G$ and $\rho^{\prime}(b)=\left(\begin{array}{cc}1 & u(b) \\ 0 & E_{m}\end{array}\right)$ for any element $b$ of $R^{u}$. Put $G^{\prime}=\rho^{\prime}(G)$ and $\bar{G}=\rho(G)$. If $v \neq 0$ on $R^{u}$, then $\bar{G}$ is reductive by the induction hypothesis. Let $\varphi: G^{\prime} \ni g^{\prime}=\left(\begin{array}{cc}1 & u \\ 0 & \bar{g}\end{array}\right) \backsim m \rightarrow \bar{g} \in \bar{G}$ be a canonical homomorphism. Then $\operatorname{Ker} \varphi=R^{\prime u}\left(R^{\prime u}\right.$ being the unipotent radical of $G^{\prime}$ and $R^{\prime u}=\rho^{\prime}\left(R^{u}\right)$ ) because characteristic of $k$ is zero. Thus $G^{\prime} / R^{\prime u}$ is isomorphic to $G$. By lemma 2.6, there exists a group homomorphism: $G \ni \bar{g} \leadsto \rightarrow\left(\begin{array}{cc}1 & s(\bar{g}) \\ 0 & \bar{g}\end{array}\right)$ $\in G^{\prime}$ and $s\left(\bar{g}_{1} \cdot \bar{g}_{2}\right)=s\left(\bar{g}_{2}\right)+s\left(\bar{g}_{1}\right) \cdot \bar{g}_{2}$ for every element $\bar{g}_{1}$ and $\bar{g}_{2}$ of $\bar{G}$. Let $g^{\prime}=\left(\begin{array}{cc}1 & u \\ 0 & \bar{g}\end{array}\right)$ be an elment of $G^{\prime}$.

$$
g^{\prime}=\left(\begin{array}{ll}
1 & u \\
0 & \bar{g}
\end{array}\right)=\left(\begin{array}{ll}
1 & s(\bar{g}) \\
0 & \bar{g}
\end{array}\right)\left(\begin{array}{ll}
1 & u-s(\bar{g}) \\
0 & 1
\end{array}\right),
$$

and

$$
\left(\begin{array}{ll}
1 & u-s(\bar{g}) \\
0 & 1
\end{array}\right) \text { is an element of } R^{\prime u}
$$

If $\operatorname{dim} R^{\prime u}=r$, then there is an isomorphism $\alpha: R^{\prime u} \rightarrow k^{\oplus r}$ as algebraic groups because $R^{\prime u}$ is commutative.

Let $\psi: G^{\prime} \ni g^{\prime} \longrightarrow$ Int $g^{\prime} \in \operatorname{Aut}_{2 \mathrm{lg} \cdot \mathrm{gr} \cdot}\left(R^{\prime u}\right)=\operatorname{Aut}_{2 \mathrm{lg} \cdot \mathrm{gr} \cdot}\left(k^{\oplus r}\right)$. Since characteristic of $k$ is zero, Aut alg.g1 $\cdot\left(k^{\oplus r}\right)=G L(r, k)$. Thus $\psi$ is a rational representation from $G^{\prime}$ to $G L(r, k)$ and $\operatorname{Ker} \psi$ contains $R^{\prime \prime}$. Hence we have a rational representation $\beta$ from $\bar{G}$ to $G L(r, k)$ such that the following diagram is commutative. 


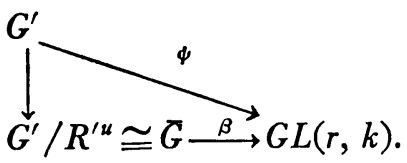

By the above, we have a rational representation $r$ from $G^{\prime}$ to $G L(r+1, k)$.

$$
r: G^{\prime} \ni g^{\prime}=\left(\begin{array}{ll}
1 & u \\
0 & \bar{g}
\end{array}\right) \longmapsto\left(\begin{array}{cc}
1 & \alpha\left(\begin{array}{cc}
1 & u-s(\bar{g}) \\
0 & 1
\end{array}\right) \\
0 & \beta(\bar{g})
\end{array}\right) \in G L(r+1, k)
$$

Then $\gamma \cdot \rho^{\prime}$ is an $M$-representation of $G$ and $r \cdot \rho^{\prime}$ has no $R^{u}$-fixed point which is not the associated fixed point. This is a contradiction. q.e.d.

Problem 2. Is a connected semi-reductive algebric group reductive?

\section{Application.}

We shall show an application of Proposition 1.8 in this section. Let $G$ be a connected algebraic group. It is well known that every invertible regular function on $G$ is a rational character up to a nonzero constant (Rosenlicht [11]). At first, we shall prove this fact directly.

Lemma 3.1. Let $T$ be a torus group. Then any invertible regular function on $T$ is a rational character up to a non-zero constant.

Proof. We can easily prove Lemma 3.1 by the induction of $\operatorname{dim} T$. q.e.p.

Definition 3.2. Let $f$ be a regular function on $G$. For an element $g$ of $G$, we define ${ }^{g} f$ (or $f^{g}$ respectively) to be $\left({ }^{g} f\right)\left(g^{\prime}\right)=f\left(g^{\prime} g\right)$ (or $f^{g}\left(g^{\prime}\right)=f\left(g^{-1} g^{\prime}\right)$ respectively) for any element $g^{\prime}$ of $G$.

Lemma 3.3. Let $G$ be a connected semi-simple algebraic group. 
Then any invertible regular function on $G$ is a non-zero constant.

Proof. Let $B, T$ and $B_{-}$be a Borel subgroup, a maximal torus of $G$ contained in $B$ and the opposite Borel subgroup of $B$ respectively. Put

$$
\varphi: B_{-}^{u} \times T \times B^{u} \ni\left(b^{\prime}, t, b\right) \mapsto \rightarrow b^{\prime} \cdot t \cdot b \in G .
$$

Then $\varphi$ is a morphism and $\operatorname{Im} \varphi$ is an affine open subset of $G$. Let $\varphi^{*}: k[G] \leftrightarrow m \rightarrow k\left[B_{-}^{u} \times T \times B^{u}\right]$ be the induced injective homomorphism. If $\operatorname{dim} B^{u}=n$, then $k\left[B_{-}^{u} \times T \times B^{u}\right]=k[T]\left[X_{1},,, X_{n}, Y_{1},,, Y_{n}\right]$, where $X_{i}$ and $Y_{i}$ are indeterminates over $k[T]$. Therefore, if $f$ is an invertible regular function on $G$, then $\varphi^{*}(f)$ is an invertible element of $k[T]$. In particular, ${ }^{b} f^{b \prime}=f$ for any element $b$ (or $b^{\prime}$ ) of $B^{u}$ (or $B_{-}^{u}$ respectively). We may assume $f(e)=1$ where $e$ is the unit element of $G$, in order to prove that $f$ is a non-zero constant. If we restrict $f$ on $T$, then it is a rational character on $T$ by virtue of Lemma 3.1. Put $Q$ to be the connected component of $\operatorname{Ker}(f \mid T)$ at e. For any element $b$ of $B^{u}, b^{\prime}$ of $B_{-}^{u}$ and $t$ of $T,{ }^{t b} f^{b \prime}=f$. Let $\sum=\left\{\alpha_{1},,, \alpha_{r}\right\}$ be a fundamental root system of $G$ with respect to $(B, T), G_{\alpha_{i}}$ the root subgroup of $G$ associated with $\alpha_{i}\left(G_{\alpha_{i}}\right.$ being a connected semi-simple subgroup and $\operatorname{dim} G_{\alpha_{i}}=3$ ), and $P_{\alpha_{i}}$ (or $P_{-\alpha_{i}}$ be a one-parameter subgroup

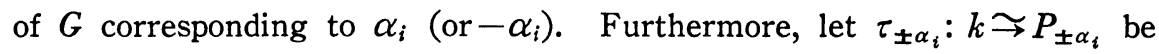
the isomorphisms and $T_{\alpha_{i}}$ be a maximal torus of $G_{\alpha_{i}}\left(\operatorname{dim} T_{\alpha_{i}}=1\right)$. Then,

(1) $T=T_{\alpha_{1}} \ldots T_{\alpha_{r}}$

(2) $P_{-\alpha_{i}} \cdot \tau_{\alpha_{i}}(1) \cdot P_{-\alpha_{i}} \cdot \tau_{\alpha_{i}}(-1) \cdot P_{\alpha_{i}}$ contains $T_{\alpha_{i}}$.

Therefore, any element $g$ of $\operatorname{Im} \varphi$ can be written in the following way;

$$
g=b^{\prime} \cdot \tau_{\alpha_{i}}(1) \tau_{-\alpha_{i}}(x) \tau_{\alpha_{i}}(-1) \cdot t \cdot b
$$

where $b^{\prime} \in B_{-}^{u}, b \in B^{u}, t \in Q$ and $x \in k$. Thus $f(g)=f\left(b^{\prime} \tau_{\alpha_{i}}(1) \tau_{-\alpha_{i}}(x)\right.$ $\left.\tau_{\alpha_{i}}(-1) t \cdot b\right)=f\left(\tau_{\alpha_{i}}(1) \tau_{-\alpha_{i}}(x) \tau_{\alpha_{i}}(1)\right)$. But it is obvious that an invertible regular function on $P_{-\alpha_{i}}$ is a non-zero constant. Hence $f$ is a non-zero constant. 
Now we can show the following Theorem 3.4.

Theorem 3.4. Let $G$ be a connected algebraic group. Then any invertible regular function on $G$ is a rational character of $G$ up to a non-zero constant.

Proof. Let $R$ be the radical of $G$ and $G^{\prime}=G / R^{u}$, where $R^{u}$ is the unipotent part of $R$. Then $G \simeq R^{u} \times G^{\prime}$ as algebraic varieties (Rosenlicht [10], Grothendieck [4]). Thus, if $n=\operatorname{dim} R^{u}$, then we have that $k[G]$ $=k\left[G^{\prime}\right]\left[X_{1}, \ldots, X_{n}\right]$, where $X_{i}$ are indeterminates over $k\left[G^{\prime}\right]$. Hence we may assume that $G$ is a reductive algebraic group in order to prove Theorem 3.4. Then $R$ is a central torus subgroup of $G, G=R[G, G]$ and $[G, G]$ is a semi-simple algebraic group. Put $\varphi: R \times[G, G]$ $\ni(b, g): m \rightarrow b \cdot g \in G$. Let $\varphi^{*}: k[G]-k[R \times[G, G]]$ be the injective homomorphism induced by $\varphi$. If $f$ is any invertible regular function on $G$, then $\varphi^{*}(f)$ is an invertible element of $k[R]$ by virtue of Lemma 3.3. Thus, $f$ is a rational character of $G$ up to a non-zero constant.

q.e.d.

Corollary 3.5. Let $G$ be a connected algebraic group whose unipotent radical is trivial. Then any invertible regular function on $G$ is a non-zero constant.

Proof. It is obvious.

Next, we shall show an application of Proposition 1.8 .

Proposition 3.6. Let $G$ be a semi-reductive algebraic group and $f$ be a non-constant regular function on $G$. Then $V=\sum_{g \in G} f^{g} \cdot k\left(\right.$ or $\left.\sum_{g \in G}^{g} f \cdot k\right)$ has a nonconstant Borel semi-invariant function on $G$.

Proof. If $V=\sum_{g \in G} f^{g} \cdot k$ has no constant function, then Proposition 3.6 is true by virtue by virtue of Lie-Kolchin's theorem. We may 
assume that $V$ has a non-zero constant function. Let $\left\{e_{0}=1, e_{1}, \ldots, e_{n}\right\}$ be a basis of $V$. Then we have an $M$-representation of $G$. If we write $x=x_{0} e_{0}+x_{1} e_{1}+\cdots+x_{n} e_{n}\left(x_{i} \in k\right)$, then $x$ is a constant function, if and only if $x_{1}=\cdots=x_{n}=0$. By virtue of Lemma 1.3, we have a Borel semi-invariant point $x=x_{0} e_{0}+x_{1} e_{1}+\cdots+x_{n} e_{n}$ where some $x_{i}$ $\neq 0(1 \leq i \leq n)$. Therefore, $V$ has a non-constant Borel semi-invariant regular function on $G$.

q.e.d.

Theorem 3.7. Let $G$ be a connected semi-simple algebraic group and $f$ be a non-constant regular function on $G$. Then $V=\sum_{g \in G} f^{g} k$ (or $\left.\sum_{g \in G}^{g} f k\right)$ has a non-invertible Borel semi-invariant regular function on $G$.

Proof. It is obvious from Proposition 1.8, Proposition 3.6 and Lemma 3.3.

q.e.d.

Remark 3.8. Unfortunately, in Theorem 3.7 we can not say the following. There exist finite elements $\left\{g_{1}, \ldots, g_{n}\right\}$ of $G$ and finite elements $\left\{x_{1}, \ldots, x_{n}\right\}$ of $k$ such that

(1) $\left\{f^{g_{1}}, \ldots, f^{g_{n}}\right\}$ is a bassi of $V$.

(2) $\sum_{i=1}^{n} x_{i} f^{g i}$ is a non-constant Borel semi-invariant regular function on $G$.

(3) $\sum_{i=1}^{n} x_{i} \neq 0$.

In fact, we can easily make a counter example.

Department of Applied Mathematics

KŌNAN UNIVERSITY

\section{Bibliograhpy}

[1] A. Borel, Groupes linéaires algébriques, Annals of Mathematics, Vol. 64, 1959.

[2] B. Birula, On homogeneous affine spaces of linear algebraic groups, Amer. Jour. of Math., Vol. 85, 1963, 577-582.

[3] C. Chevalley, Classification des groupes de Lie algébriques, Séminaire C. 
Chevalley, 1956-1958.

[4] C. Chevalley, Certains schémas de groupes semi-simples, Séminaire Bourbaki, 1960-61.

[5] A. Grothendieck, Torsion homologique et sections rationnelles, Séminaire C. Chevalley, 1958.

[6] D. Mostow, Fully reducible subgroups of algebraic groups, Amer, Jour. of Math., Vol. 78, 1956, 200-221.

[7] D. Mumford, Geometric invariant theory, Springer Band 34, 1965.

[8] D. Mumford, Introduction to algebraic geometry, Lecture note at Harvard Univ.

[9] M. Nagata, Invariants of a group in an affine ring, Jour. of Math. Kyoto Univ., Vol. 3, 1964, 369-377.

[10] M. Nagata and T. Miyata, Note on semi-reductive groups, Jour. of Math. Kyoto Univ., Vol. 3, 1964, 379-382.

[11] M. Nagata, Lectures on the fourteenth problem of Hilbert, Tata Institute, 1965.

[12] M. Rosenlicht, Some basic theorems on algebraic groups, Amer. Jour. of Math. Vol. 78, 1956, 401-443.

[13] M. Rosenlicht, Toroidal algebraic groups, Proc. Amer. Math. Soc., Vol. 12, 1961, 984-988.

[14] C. Seshadri, On Mumford conjecture for GL(2) and applications. Algebraic geometry, Tata Institute, 1969.

[15] R. Steinberg, Gènèrateurs, rélations et revetements de groupes algébriques, Colloque sur la théorie des groupes algébriques, Brussels, 1962. 